\title{
Efeito da co-dopagem com Ce, Tb e Mn nas propriedades morfológica, estrutural e ótica de filmes de alumina
}

\section{Effect of co-doping with $\mathrm{Ce}, \mathrm{Tb}$ and $\mathrm{Mn}$ on the morphological, structural and optical properties of alumina films}

Daniele Fontes Henrique ${ }^{1,2}$, Herval Ramos Paes Junior ${ }^{2}$

\author{
${ }^{1}$ Instituto Federal de Educação, Ciência e Tecnologia Fluminense - IFF \\ Rua Amilcar Pereira da Silva 727, Piteiras, Quissamã - RJ \\ e-mail:danielefhenrique@gmail.com \\ ${ }^{2}$ Laboratório de Materiais Avançados/CCT- Universidade Estadual do Norte Fluminense Darcy Ribeiro - UENF \\ Av. Alberto Lamego, 2000, Parque California - Campos dos Goytacazes - RJ \\ e-mail:hervalpaes@gmail.com
}

\section{RESUMO}

O sistema de iluminação vem sofrendo algumas transformações no decorrer dos anos. Atualmente o sistema a LED (Light Emitting Diode - diodo emissor de luz) tem se destacado, mostrando que tem potencial para emitir luz de forma mais eficiente e ainda reduzindo o consumo de energia elétrica. O objetivo principal deste trabalho foi a análise da fotoluminescência dos filmes produzidos utilizando a técnica de deposição spraypirólise que tem como característica ser um sistema simples, de baixo custo e eficiente. Além disso, foram investigados os efeitos da co-dopagem com cério, térbio e manganês nas propriedades morfológicas, estruturais e óticas de filmes de alumina. Foi analisada a influência da temperatura de substrato, que variou entre 300 e $450{ }^{\circ} \mathrm{C}$, sobre os espectros de absorbância, transmitância e emissão fotoluminescente para filmes depositados por spray-pirólise sobre substratos de vidro. O fluxo da solução precursora adotado foi de $0,5 \mathrm{~mL} / \mathrm{min}$ e o tempo de deposição de 3 minutos. Através da co-dopagem com $5 \%$ at. de $\mathrm{Ce}, 1 \%$ at. de Tb e $1 \%$ at. de Mn nos filmes de alumina foi possível obter a emissão de luz branca. A melhor condição de emissão luminescente foi a amostra depositada em $300^{\circ} \mathrm{C}$. A deposição por spray-pirólise mostrou-se eficiente na obtenção de filmes fotoluminescentes, confirmando a capacidade para aplicações em sistemas de iluminação eficientes, como por exemplo, em w-LED (White Light Emitting Diode - diodo emissor de luz branca).

Palavras-chave: Fotoluminescência, alumina, spray-pirólise, diodo emissor de luz branca.

\section{ABSTRACT}

The lighting system has undergone some changes over the years. Currently the LED (Light Emitting Diode) system has stood out, showing that it has the potential to emit light more efficiently and still reducing the consumption of electric energy. The main objective of this work was the analysis of the photoluminescence of the films produced using the spray-pyrolysis deposition technique, which has the characteristic of being a simple, low cost and efficient system. In addition, the effects of co-doping with cerium, terbium and manganese on the morphological, structural and optical properties of alumina films were investigated. The influence of the substrate temperature, which varied between 300 and $450{ }^{\circ} \mathrm{C}$, on the absorbance, transmittance and photoluminescent emission spectra for films deposited by spray-pyrolysis on glass substrates was analyzed. The precursor solution flow was set at $0.5 \mathrm{~mL} / \mathrm{min}$ and the deposition time of 3 minutes. By co-doping with $\mathrm{Ce}$ (5 at.\%), Tb (1 at.\%) and Mn (1 at.\%) in alumina films was possible to obtain white light emission. The best luminescent emission condition was the sample deposited at $300{ }^{\circ} \mathrm{C}$. The spray pyrolysis deposition technique proved effective in obtaining photoluminescent films, confirming the ability for applications in efficient lighting systems, such as w-LED (white light emitting diode). 
Keywords: Photoluminescence, alumina, spray pyrolysis, w-LED.

\section{INTRODUÇÃO}

Muitas pesquisas são realizadas na área de filmes com o intuito de obter melhores resultados e aplicações diversas como em "displays" planos, iluminação, catalisadores, pilha combustível, etc. Nos últimos anos as iluminações a LED (Light Emitting Diode -Diodo Emissor de Luz) tem despertado o interesse da indústria e também atraído consumidores. O uso de diodos emissores de luz branca (w-LEDs) vem se apresentando como fonte de iluminação de alta intensidade, além de apresentar vantagens como longevidade, tamanho reduzido e, principalmente, baixo consumo de energia [1,2]. Existem diferentes técnicas de deposição de filmes como, por exemplo, a pulverização catódica (sputtering), sol-gel e spray-pirólise. Das técnicas citadas é relatado na literatura que o método spray-pirólise apresenta algumas características como: alta eficiência, baixo custo e simplicidade [3]. Em função destas características esta técnica foi adotada no presente trabalho.

$\mathrm{O}$ filme de óxido de alumínio $\left(\mathrm{Al}_{2} \mathrm{O}_{3}\right)$, também chamado de alumina, é a rede hospedeira que pode incorporar íons de cério $(\mathrm{Ce})$, térbio $(\mathrm{Tb})$ e manganês $(\mathrm{Mn})$. Estes são os ativadores que produzem emissões luminescentes em determinados comprimentos de ondas que correspondem a uma cor quando excitados por uma fonte de energia, ultravioleta ou raios X. Esse efeito é chamado de fotoluminescência.

A alumina apresenta características interessantes como: resistência a temperaturas elevadas, elevada rigidez e alta estabilidade química [4,5]. Por isso, é considerado um bom material hospedeiro. O Ce é um dos íons terras raras que possui emissão na faixa do azule violeta $[4,6,7]$. O Tb é uma das terras raras que pode apresentar fotoluminescência na faixa do azul e do verde simultaneamente [6,7]. Todavia, para ser observada a luminescência destes íons devem apresentar-se na forma trivalente e, portanto, tanto o material precursor quanto às condições de produção e de processamento dos filmes devem ser criteriosamente avaliadas. $\mathrm{O} \mathrm{Mn}$ é um metal em transição que apresenta fotoluminescência na cor vermelha $[7,8]$.

A combinação desses três íons na rede hospedeira $\mathrm{Al}_{2} \mathrm{O}_{3}$ trouxe resultados interessantes para aplicação na tecnologia de iluminação com LED branco (w-LED), na qual a amostra emitiu luz branca quando submetida ao espectro ultravioleta.

\section{MATERIAIS E MÉTODOS}

Os filmes de alumina co-dopados com Ce, $\mathrm{Tb}$ e $\mathrm{Mn}$ foram depositados por spray-pirólise mecânico sobre substratos de vidro comum. Foram preparadas soluções precursoras a partir da mistura de 24,143 g de cloreto de alumínio hexahidratado $\left(\mathrm{AlCl}_{3} \cdot 6 \mathrm{H}_{2} \mathrm{O}\right)$ com três partes de álcool isopropílico e uma parte de água deionizada na concentração de $0,1 \mathrm{M}$. A mesma concentração foi utilizada para cada elemento dopante: $5 \%$ at. de cloreto de cério heptahidratado $\left(\mathrm{CeCl}_{3} .7 \mathrm{H}_{2} \mathrm{O}\right)$ com $99,9 \%$ de pureza, $1 \%$ at. de cloreto de térbio hexahidratado $\left(\mathrm{TbCl}_{3} \cdot 6 \mathrm{H}_{2} \mathrm{O}\right)$ com $99,9 \%$ de pureza e $1 \%$ at. de cloreto de manganês hexahidratado $\left(\mathrm{MnCl}_{2} \cdot 6 \mathrm{H}_{2} \mathrm{O}\right)$ com $99 \%$ de pureza.

Foram realizadas deposições nas temperaturas do substrato em $300,350,400$ e $450^{\circ} \mathrm{C}$ com duração de 3 minutos e o fluxo da solução precursora foi de $0,5 \mathrm{~mL} / \mathrm{min}$. A análise da estrutura cristalina dos filmes foi feita com o difratômetro de raios X (DRX) modelo SHIMADZU XRD - 7000, utilizando o comprimento de onda de $1,54 \AA$ (radiação $\mathrm{CuK} \alpha$ ), passo de varredura de $0,02^{\circ}$ e tempo por passo de 1 segundo. Para a observação e análise da superfície e da microestrutura do material foi realizada a caracterização morfológica com o auxílio do microscópio confocal a laser modelo OLS4000 fabricado pela Olympus. Através das micrografias da seç̧ão transversal, obtidas pelo microscópio confocal, foi possível medir as espessuras dos filmes. Para realizar a caraterização ótica utilizou-se o espectrofotômetro Shimadzu modelo UV-1800 para analisar a transmissão e absorção do filme em função do comprimento de onda da luz e o espectrofluorímetro RF5301PC da Shimadzu que mede a fotoluminescência emitida pelo filme em função do comprimento de onda sobre a amostra.

\section{RESULTADOS}

\subsection{Caracterização Estrutural}

A Figura 1 mostra os difratogramas dos filmes de $\mathrm{Al}_{2} \mathrm{O}_{3}$ co-dopados e depositados nas temperaturas de $300 \mathrm{e}$ $400^{\circ} \mathrm{C}$. Os filmes apresentam uma estrutura cristalina com a identificação de duas fases. Observa-se que os ângulos de difração são os mesmos ou bem próximos. Entretanto, ocorre uma redução na intensidade dos picos quando depositados em temperaturas mais altas. Com o auxílio do JCPDS arquivo PDF\#70-2038 a identificação dos quatros primeiros picos da varredura com ângulo $2 \theta$ de $37,62^{\circ}, 44,09^{\circ}, 64,54^{\circ}$ e $77,59^{\circ}$ correspondem a fase da gibsita $\mathrm{Al}(\mathrm{OH})_{3}$. Os três últimos picos de menor intensidade não são relacionados nesse 
arquivo. Entretanto, o arquivo PDF\#03-0146, que é referente ao óxido de alumínio hidratado $\alpha-\mathrm{Al}(\mathrm{OH})_{3}$, complementa a identificação dos picos observados no ângulo $2 \theta$ de $98,08^{\circ}, 111,65^{\circ}$ e $115,45^{\circ}$.
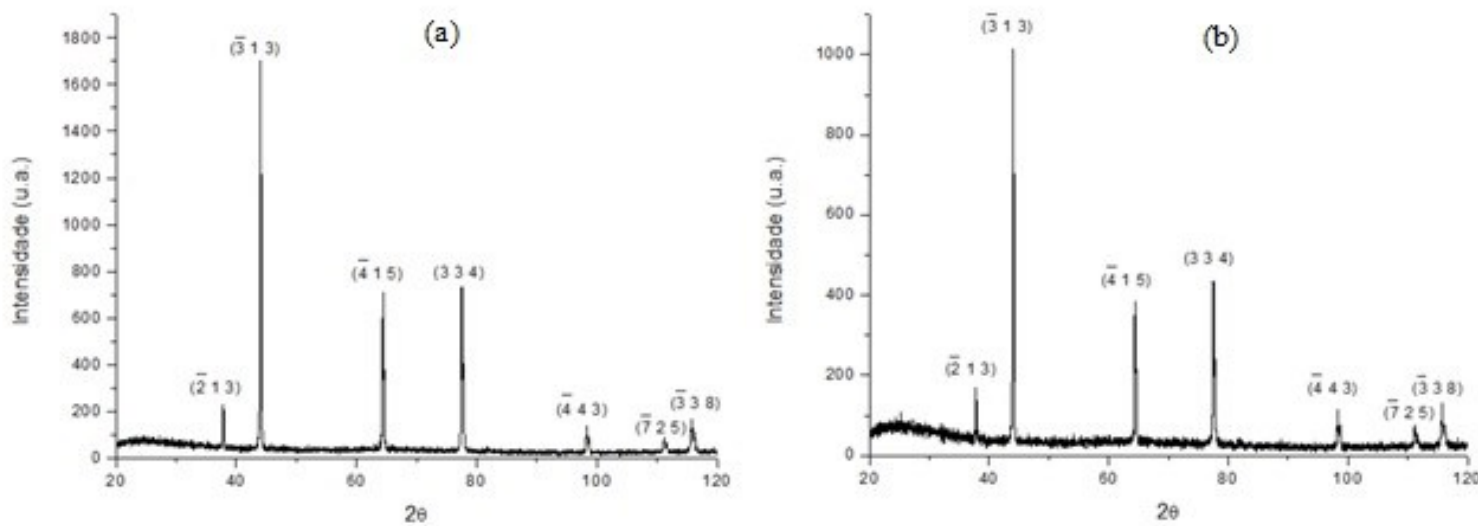

Figura 1: Difratogramas de raios $\mathrm{X}$ dos filmes de alumina co-dopados com Ce, $\mathrm{Tb}$ e $\mathrm{Mn}$ depositados em (a) $300^{\circ} \mathrm{C}$ e (b) $400^{\circ} \mathrm{C}$. Os primeiros quatro picos correspondem a formação de $\mathrm{Al}(\mathrm{OH})_{3}$ e os três últimos pequenos picos referem-se à $\alpha$ $\mathrm{Al}(\mathrm{OH})_{3}$.

A gibsita é um material precursor da alumina. À medida que a temperatura é elevada o hidróxido vai perdendo moléculas de água ocasionando transformações e passagens de fase até alcançar a sua fase mais estável, a alfa alumina $\left(\alpha-\mathrm{Al}_{2} \mathrm{O}_{3}\right)$. Nessas transformações há a formação de hidróxido $\left(\mathrm{Al}(\mathrm{OH})_{3}\right)$ e os oxidróxidos de alumínio $(\mathrm{AlOOH})$.

Através dos picos mais intensos foram realizados os cálculos do tamanho dos cristalitos dos filmes da Figura 1 com a utilização da equação de Scherrer. Com o uso de um software específico foi possível encontrar os valores da largura de pico a meia altura $(B \mathrm{hkl})$ e o ângulo de difração de Bragg $(\theta)$ e traçar a gaussiana do pico mais intenso de cada amostra. Com as curvas gaussianas foi possível estimar o tamanho dos cristalitos que ambos apresentaram aproximadamente $45,12 \mathrm{~nm}$, como observa-se na Tabela 1 . Ou seja, não houve uma alteração significativa entre os filmes.

Tabela 1: Tamanho de cristalito para filmes de alumina

\begin{tabular}{c|c|c}
\hline Filmes de $\mathrm{Al}_{\mathbf{2}} \mathbf{O}_{\mathbf{3}}$ & $\begin{array}{c}\text { Temperatura de } \\
\text { deposição }\left({ }^{\circ} \mathbf{C}\right)\end{array}$ & $\begin{array}{c}\text { Diâmetro do } \\
\text { cristalito (nm) }\end{array}$ \\
\hline Intrínseco & 300 & 43,56 \\
\hline $\begin{array}{c}\text { Co-dopado com 5\% at. de Ce, } \\
\text { 1\% at. de Tb e 1\% at. de Mn }\end{array}$ & 300 & 45,12 \\
\cline { 2 - 3 } & 400 & 45,12 \\
\hline
\end{tabular}

\subsection{Caracterização Morfológica}

Ao fixar o fluxo de solução em $0,5 \mathrm{~mL} / \mathrm{min}$ e variar a temperatura de deposição, verifica-se que os filmes de alumina co-dopados com $\mathrm{Ce}, \mathrm{Tb}$ e $\mathrm{Mn}$ apresentaram trincas que diminuem a medida que aumenta a temperatura de deposição. Além disso, os filmes revelaram pouca aderência ao substrato de vidro, principalmente nas temperaturas mais baixas $\left(300\right.$ e $\left.350^{\circ} \mathrm{C}\right)$. Essas características podem ser acompanhadas na Figura 2.
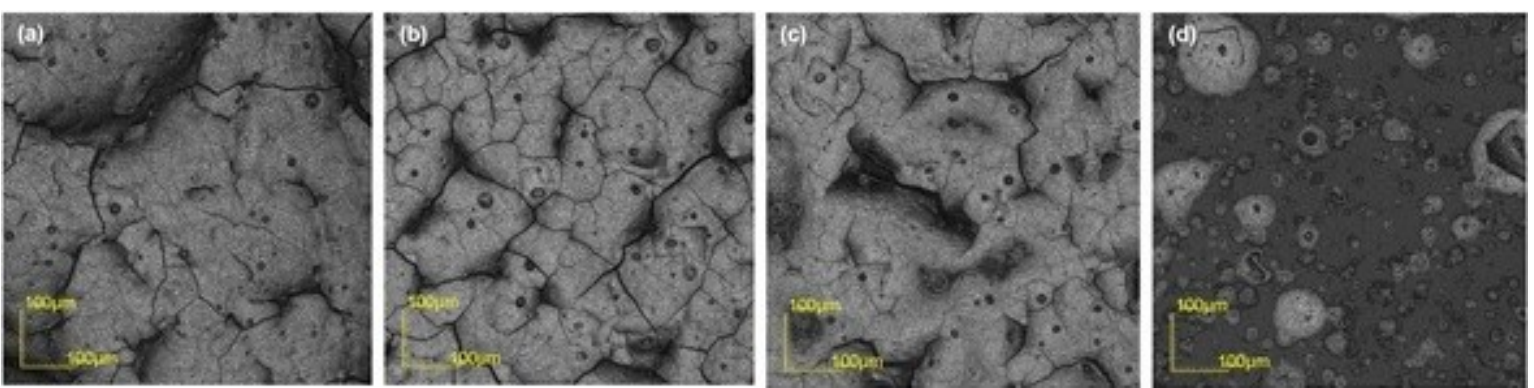

Figura 2: Micrografias de filmes de $\mathrm{Al}_{2} \mathrm{O}_{3}$ co-dopados com $5 \%$ at. de $\mathrm{Ce}, 1 \%$ at. de $\mathrm{Tb}$ e $1 \%$ at. de $\mathrm{Mn}$, depositados em (a) $300^{\circ} \mathrm{C}$, (b) $350^{\circ} \mathrm{C}$, (c) $400^{\circ} \mathrm{C}$ e (d) $450^{\circ} \mathrm{C}$. Aumento de $430 \mathrm{X}$.

Quando depositado em $450^{\circ} \mathrm{C}$ a solução evapora mais rapidamente antes de chegar à superfície do 
substrato, por isso alguns pontos não há a presença de filme, revelando uma não continuidade do filme sobre o substrato. A técnica spray-pirólise é uma variante da deposição química em fase vapor (CVD). As gotículas da solução que saem do spray em direção à superfície do substrato devem passar pelo processo da evaporação de modo que somente o precursor, sem o solvente, alcance a superfície do substrato na fase gasosa, ocorrendo a reação química sobre o substrato formando desta forma o filme. Porém, o processo nem sempre acontece dessa maneira, pois as gotículas saem do bico atomizador com tamanhos diferentes, consequentemente algumas gotas evaporam antes e outras depois de chegarem sobre a superfície. Isso implica de forma negativa na qualidade do filme, pois possibilitará a formação de trincas, superfície rugosa e baixa aderência ao substrato. Os filmes apresentaram espessuras de 5,52 $\mu \mathrm{m}, 4,66 \mu \mathrm{m}, 3,77 \mu \mathrm{m}$ e $2,77 \mu \mathrm{m}$ quando depositados em $300,350,400$ e $450^{\circ} \mathrm{C}$, respectivamente.

\subsection{Caracterização Ótica}

Na Figura 3 são apresentadas as curvas da variação da absorção e transmitância em função do comprimento de onda para os filmes de alumina co-dopados com $5 \%$ at. de cério, $1 \%$ at. de térbio e $1 \%$ at. de manganês, depositados nas temperaturas de $300,350,400 \mathrm{e} 450^{\circ} \mathrm{C}$. Nota-se que a absorção é bem menor na temperatura de $450^{\circ} \mathrm{C}$, já que a espessura do filme é bem menor que a das outras amostras. Observa-se que o filme depositado a $350^{\circ} \mathrm{C}$ apresenta maior absorção em $300 \mathrm{~nm}$, mas na região do comprimento de onda do visível, ou seja, aproximadamente de 400 a $700 \mathrm{~nm}$ o filme que mais absorve é o que foi depositado em $300^{\circ} \mathrm{C}$.
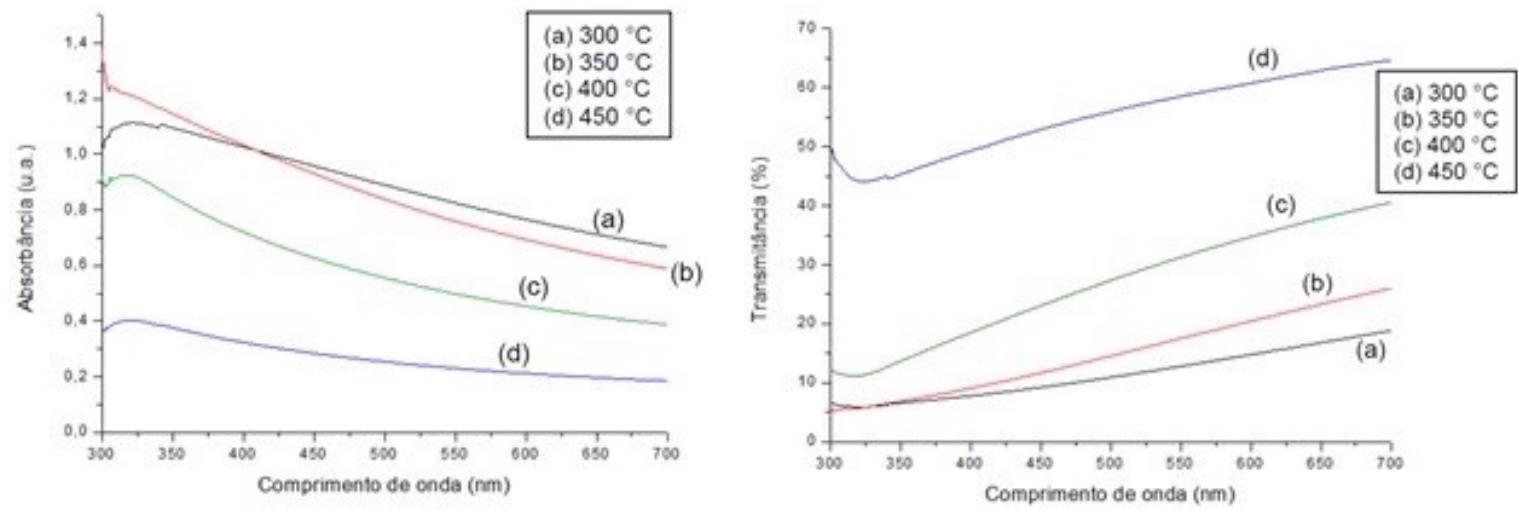

Figura 3: Curva da variação da absorção e transmitância ótica em função do comprimento de onda para filmes de alumina co-dopados com $5 \%$ at. de Ce, $1 \%$ at. de Tb e $1 \%$ at. de Mn depositados em 300, 350, 400 e $450^{\circ} \mathrm{C}$.

De todas as amostras que foram realizadas as medidas de transmitância, o filme de alumina co-dopado depositado a $450^{\circ} \mathrm{C}$ foi o único que apresentou transmitância acima de $50 \%$. A transmitância da amostra depositada a $300^{\circ} \mathrm{C}$ ficou abaixo de $20 \%$, valor que é considerado bom para a produção de filmes fotoluminescentes.

Os espectros de emissão fotoluminescente para filmes de alumina co-dopados com Ce, $\mathrm{Tb}$ e $\mathrm{Mn}$ são mostrados na Figura 4 quando excitados pelo comprimento de onda de 300nm. Quatro picos se destacam na figura. Os picos estão centrados em $363 \mathrm{~nm}, 437 \mathrm{~nm}, 548 \mathrm{~nm}$ e $614 \mathrm{~nm}$. O terceiro pico provavelmente é influenciado pela presença do térbio na solução [7]. Em óxidos co-dopados o $\mathrm{Ce}^{3+}$ pode atuar como um bom sensibilizador ao transferir parte da energia para ativar íons terra rara trivalente [9]. Outro fato interessante a ser notado é que no primeiro pico observa-se uma queda brusca na intensidade da luminescência quando a temperatura de deposição é aumentada. Isto, provavelmente está associado à alteração de valência do cério [7]. À medida que aumenta o comprimento de onda a diferença na intensidade de emissão diminui. 


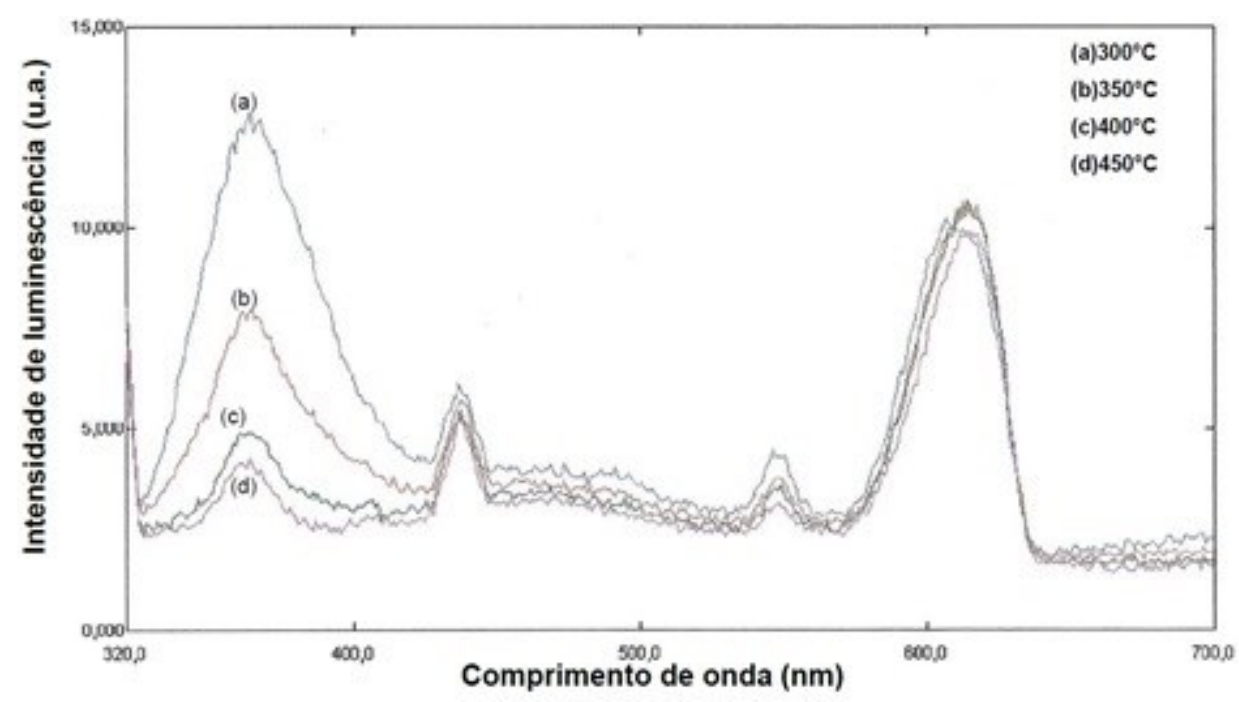

Figura 4: Espectros de emissão de filmes de alumina co-dopados com 5\% at. de Ce, $1 \%$ at. de Tb e 1\% at. de Mn depositados em $300,350,400$ e $450^{\circ} \mathrm{C}$.

Os filmes de alumina dopados separadamente pelos elementos cério, térbio e manganês emitiram luz na cor azul, azul-esverdeado e vermelho, respectivamente. Sabe-se que com as cores primárias, azul, verde e vermelho obtém-se a cor branca. Ao produzir amostras de alumina co-dopados com 5\% at. de cério, $1 \%$ at. de térbio e $1 \%$ at. de manganês foi possível obter a luz branca, Figura 5, quando submetido ao comprimento de onda na faixa do ultravioleta $300 \mathrm{~nm}$.

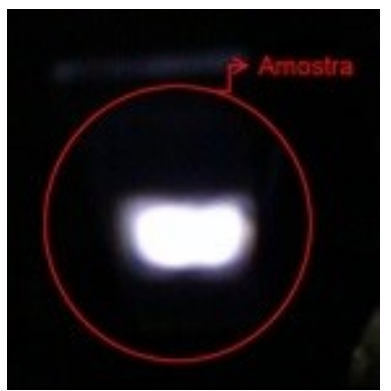

Figura 5: Amostra do filme de alumina co-dopado 5\% at. de $\mathrm{Ce}, 1 \%$ at. de $\mathrm{Tb}$ e $1 \%$ at. de $\mathrm{Mn}$ depositado na temperatura de $300^{\circ} \mathrm{C}$ no espectrofluorímetro sendo excitado em $300 \mathrm{~nm}$ e irradiando luz branca.

\section{CONCLUSÕES}

Os filmes de alumina co-dopados com Ce, Tb e Mn apresentaram estrutura cristalina compatível com a gibsita. Os filmes apresentaram trincas e pouca aderência ao substrato. Considerando que o objetivo desse trabalho é de analisar a fotoluminescência dos filmes de alumina co-dopados a amostra produzida a $300{ }^{\circ} \mathrm{C}$ foi que apresentou melhor absorção do ultravioleta, consequentemente menor porcentagem de transmitância, destacando-se com a emissão luminescente de maior intensidade no comprimento de onda da luz visível. Com a co-dopagem dos filmes de $\mathrm{Al}_{2} \mathrm{O}_{3}$ com 5\% at. de Ce, 1\% at. de $\mathrm{Tb}$ e $1 \%$ at. de $\mathrm{Mn}$ foi possível obter a fotoluminescência emitindo luz branca, demonstrando a viabilidade da aplicação da técnica de spray-pirólise para produção de materiais para sistemas de iluminação como, por exemplo, w-LED.

\section{AGRADECIMENTO}

Ao CNPQ pelo apoio financeiro. 


\section{BIBLIOGRAFIA}

[1] CALDiÑO, U., ÁlvarEZ, E., SPEGHINI, A., et al., "Cold and warm white light generation using $\mathrm{Zn}\left(\mathrm{PO}_{3}\right)_{2}$ glsses activated by $\mathrm{Ce}^{3+}$ and $\mathrm{Mn}^{2+}$, Journal of Luminescence, v.132, pp. 2077-2081, Aug. 2012.

[2] ÁlVAREZ, E., ZAYAS, MA.E., RODRIGUEZ-CARVAJAL, D., et al., "Cold White light geberation through the simultaneous emission from $\mathrm{Ce}^{3+}$ and $\mathrm{Tb}^{3+}$ in sodium germinate glass", Optical Materials, v. 37, pp. 451-456, Nov. 2014.

[3] DHONGE, B.P., MATHEUS, T., SUNDARI, S.T., et al., "Spray pyrolytic deposition of transparent aluminum oxide $\left(\mathrm{Al}_{2} \mathrm{O}_{3}\right)$ films", Applied Surface Science, v. 258, pp. 1091-1096, Nov. 2011.

[4] MARTÍNEZ-MARTÍNEZ, R., RVERA, S., YESCAS-MENDOZA, E., et al., "Luminescence properties of $\mathrm{Ce}^{3+}-\mathrm{Dy}^{3+}$ codoped aluminium oxide films", Optical Materials, v. 33, pp. 1320-1324, Jun. 2011.

[5] ZHU, Z., LIU, D., LIU, H., et al., "Photoluminescence properties of $\mathrm{Tb}^{3+}$ doped $\mathrm{Al}_{2} \mathrm{O}_{3}$ microfibers via hydrothermal route followed by heat treatment", Ceramics International, v. 38, pp. 4137-4141, Jul. 2012.

[6] VIANA, C.C.B., Fotoluminescência de filmes de aluminja dopados com cério depositados por spraypirólise, Tese de D.Sc., UENF, Campos dos Goytacazes, RJ, Brasil, 2005.

[7] MARTÍNEZ-MARTÍNEZ, R., ÁLVAREZ, E., SPEGHINI, A., et al., "White light generation in $\mathrm{Al}_{2} \mathrm{O}_{3}: \mathrm{Ce}^{3+}: \mathrm{Tb}^{3+}: \mathrm{Mn}^{2+}$ films deposited by ultrasonic spray pyrolysis", Thin Solid Films, v. 518, pp. 57245730, Aug. 2010.

[8] GONZÁLEZ, W., ÁlvareZ, E., MARTíNEZ-MARTÍNEZ, R., et al., "Cold white light generation through the simultaneous emission from $\mathrm{Ce}^{+3}, \mathrm{Dy}^{+3}$ and $\mathrm{Mn}^{+2}$ in $90 \mathrm{Al}_{2} \mathrm{O}_{3} .2 \mathrm{CeCl}_{3} .3 \mathrm{DyCl}_{3} .5 \mathrm{MnCl}_{2}$ thin film", Journal of Luminescence, v. 132, pp. 2130-2134, Aug. 2012.

[9] GONZÁLEZ, W., ÁLVAREZ, E., RIGHINI, G.C., et al., "New yellowish-green light emitting thin film: $89 \mathrm{Al}_{2} \mathrm{O}_{3} .5 \mathrm{CeCl}_{3} .3 \mathrm{EuCl}_{3} .3 \mathrm{TbCl}_{3}$ ", Optical Materials, v. 35, pp. 1304-1308, Apr. 2013. 